Bangladesh J. Plant Taxon. 15(1): 67-72, 2008 (June)

(c) 2008 Bangladesh Association of Plant Taxonomists

- Short communication

\title{
MEDICO-BOTANICAL REPORT ON THE CHAKMA COMMUNITY OF BANGLADESH
}

\author{
Snigdha Roy, Mohammad Zashim Uddin ${ }^{1}$, Md. Abul Hassan \\ AND M. MATIUR RAHMAN ${ }^{2}$ \\ Department of Botany, University of Dhaka, Dhaka 1000, Bangladesh \\ Keywords: Chakma tribe, Ethnobotany, Bangladesh
}

Bangladesh is the abode for 21 ethnic communities (Khaleque 1995). Among them, the Chakma tribe is the largest and the most dominant one. Total population of Chakma is about 253,000 (Tripura 1994) of which more than 90 percent live in Rangamati and Khagrachari districts. Even in the recent past, the Chakma people living in Bangladesh used to meet their daily needs mostly from natural forest products. For the primary health care, still most of them depend upon surrounding plants and plant products. The knowledge of such health care system is passed from generation to generation in verbal form by traditional medicine men, local headmen and elderly persons in their community. However, currently the indigenous healthcare knowledge of Chakma tribe is in great risk because of various threats. If the present trend of eroding situation prevails, the valuable knowledge possessed by the Chakma people on indigenous medicinal plants is going to be lost forever without being properly recorded and documented.

Studies on medico-botanical information of ethnic communities in Bangladesh are at initial stage. Some of the articles published in this field include Mia and Huq (1988), Alam (1992), Alam et al. (1996), Khisa, B. (1996), Khisa, S.K. (1998), Rahman and Uddin (1998), Rahman et al. (1998), Uddin (2001), Uddin et al. (2001), Khan et al. (2002), Yusuf et al. (2002), Chakma et al. (2003), Rahman (2003) and Uddin et al. (2004, 2006). None of these articles cover the entire medico-botanical documentation of the Chakma people of Bangladesh. In order to address this issue, the present article attempts to present some new medico-botanical information of Chakma people in the Chittagong Hill Tracts of Bangladesh.

Rangamati and Khagrachari districts (latitude $21^{\circ} 91^{\prime}-23^{\circ} 75^{\prime} \mathrm{N}$ and longitude $91^{\circ} 75^{\prime}$ $92^{\circ} 42^{\prime}$ E) were selected for the study as the majority of the Chakma community live there. Six field trips were conducted in the study area in the years 2004 and 2006. Information on the medicinal plants was gathered by interviewing Chakma traditional medicine men, local headmen and elderly persons in the community. Local names of each medicinal plant with plant part(s) used and the names of diseases or symptoms treated were recorded. This information was confirmed by asking two or more persons of the same community. The collected botanical specimens were identified at the Department of Botany of the University of Dhaka and Bangladesh National Herbarium. The voucher specimens are stored at Bangladesh National Herbarium for future reference.

${ }^{1}$ Corresponding author. E-mail: zashim07@yahoo.com

${ }^{2}$ Present address: House 64, Road 9A, Dhanmondi R/A, Dhaka 1205, Bangladesh. 
A total of 90 plant species have been recorded which are used in the treatment of different ailments by the Chakma people. For each species, scientific, local and family names, part(s) used and diseases treated are presented in the Table 1. Out of the total 90 species, three species, viz. Brownea coccinea Jacq., Gomphostemma parvifloria Wall. and Pyrrosia piloselloides M.G. (Pteridophyte) were recorded to have medicinal value for the first time from Bangladesh (Hassan and Khan 1986, 1996, Mia and Huq 1988, Alam 1992, Alam et al. 1996, Yusuf et al. 1994, 2006, Chowdhury et al. 1996, Ghani 1998, Uddin et al. 2001, 2004, 2006, Khan et al. 2002, Chakma et al. 2003, Rahman et al. 2003). The list of medicinal plants of Chakma people presented in this article is not a complete list. To make a complete list further long term survey is necessary.

Table 1. A list of medicinal plants used by the Chakma people of Bangladesh. Scientific names of medicinal plants are arranged in alphabetical order.

\begin{tabular}{|c|c|c|c|c|c|}
\hline $\begin{array}{l}\text { Sl. } \\
\text { No. }\end{array}$ & Scientific name & Chakma name & Family & Part(s) used & $\begin{array}{l}\text { Disease(s) or symptom(s) } \\
\text { to be treated }\end{array}$ \\
\hline 1. & $\begin{array}{l}\text { Abelmoschus moschatus } \\
\text { Medik. }\end{array}$ & Kona-gach & Malvaceae & $\begin{array}{l}\text { Leaves, } \\
\text { seeds }\end{array}$ & Stomach ache \\
\hline 2. & Abroma augusta L. & Gash-chola & Sterculiaceae & Calyx, seeds & Snake bite \\
\hline 3. & Achyranthes aspera $\mathrm{L}$. & Ubo-langara & Amaranthaceae & Leaves & Stomach ache, abortion \\
\hline 4. & Adhatoda zeylanica Medik. & Basok-pata & Acanthaceae & Leaves & Cold, cough \\
\hline 5. & Ageratum conyzoides L. & $\begin{array}{l}\text { Monimozza- } \\
\text { khar }\end{array}$ & Asteraceae & Leaves & Wounds, skin diseases \\
\hline 6. & Alocasia indica (Rox.) Scott. & Man-kuchu & Araceae & Leaves & $\begin{array}{l}\text { Rheumatism, } \\
\text { constipation }\end{array}$ \\
\hline 7. & Aloe indica $\mathrm{L}$. & Ghrito-kumari & Liliaceae & Leaves & Wounds, burning \\
\hline 8. & Alpinia conchigera Griff. & Khetranga & Zingiberaceae & Rhizomes & Wounds \\
\hline 9. & Amaranthus viridis L. & Bhul-maresh & Amaranthaceae & Leaves & Fever \\
\hline 10. & $\begin{array}{l}\text { Ampelygonum chinensis (L.) } \\
\text { Lindly }\end{array}$ & Mono-eja-dar & Polygonaceae & Whole plant & Antiseptic \\
\hline 11. & Ananus sativus Schult. & Anash & Bromeliaceae & Unripe fruits & Anthelmintic \\
\hline 12. & $\begin{array}{l}\text { Angiopteris evecta (Frost.) } \\
\text { Hoffm. }\end{array}$ & Hadibo-muro & Angiopteridaceae & $\begin{array}{l}\text { Stems } \\
\text { (caudex) }\end{array}$ & Blood cancer \\
\hline 13. & Anisomelis indica (L.) Kuntze & $\begin{array}{l}\text { Jharbo-horin } \\
\text { sing }\end{array}$ & Lamiaceae & Leaves & Gout, rheumatism \\
\hline 14. & $\begin{array}{l}\text { Aphania danura (Roxb.) } \\
\text { Radlk. }\end{array}$ & Gach-challa & Sapindaceae & Roots bark & Dysentery \\
\hline 15. & Areca catechu L. & Subori & Arecaceae & Roots & Urination problem \\
\hline 16. & Azadirachta indica A. Juss. & Nim & Meliaceae & Leaves & Skin diseases \\
\hline 17. & $\begin{array}{l}\text { Baliospermum montanum } \\
\text { (Willd) Muell. }\end{array}$ & Shapan-pan & Euphorbiaceae & Leaves & Antidote \\
\hline 18. & Bambusa tulda Roxb. & Midinga-bash & Poaceae & Leaves & Diabetes \\
\hline 19. & Barleria lupulina Lindl. & Sornomukhi & Acanthaceae & Whole plant & Skin diseases \\
\hline 20. & Bombax ceiba L. & $\begin{array}{l}\text { Shimul-tuolo- } \\
\text { gach }\end{array}$ & Bombacaceae & $\begin{array}{l}\text { Roots, } \\
\text { flowers }\end{array}$ & $\begin{array}{l}\text { Impotency, pox, } \\
\text { aphrodisiac, food }\end{array}$ \\
\hline 21. & Brownea coccinea Jacq. & Kurochit-sak & Fabaceae & $\begin{array}{l}\text { Roots, } \\
\text { leaves }\end{array}$ & Gynecological problem \\
\hline 22. & Cajanus cajan (L.) Huth. & Dumisumi & Fabaceae & Leaves & Jaundice, diabetes \\
\hline
\end{tabular}


Table 1 contd.

\begin{tabular}{|c|c|c|c|c|c|}
\hline $\begin{array}{l}\text { Sl. } \\
\text { No. }\end{array}$ & Scientific name & Chakma name & Family & Part(s) used & $\begin{array}{l}\text { Disease(s) or symptom(s) } \\
\text { to be treated }\end{array}$ \\
\hline 23. & Calamus latifolius Roxb. & Karat-bet & Arecaceae & Stems & Fracture \\
\hline 24. & Calotropis gigantea $\mathrm{R}$. Br. & Akonda & Asclepiadaceae & $\begin{array}{l}\text { Leaves, } \\
\text { latex }\end{array}$ & Asthma, wounds \\
\hline 25. & $\begin{array}{l}\text { Cardiospermum helicacabum } \\
\text { L. }\end{array}$ & $\begin{array}{l}\text { Kataboksa- } \\
\text { shak }\end{array}$ & Sapindaceae & Whole plant & Measles \\
\hline 26. & Cassia hirsuta L. & Sabo-daru & Fabaceae & Leaves & Snake bite \\
\hline 27. & Celosia argentea L. & Hiang-morish & Amaranthaceae & Leaves & Ear diseases \\
\hline 28. & Celosia cristata L. & Radakuro-phul & Amaranthaceae & Leaves & Wounds \\
\hline 29. & Centella asiatica Urban. & Thankuni & Umbelliferae & Whole plant & Blood dysentery \\
\hline 30. & Centipeda minima (L.) A.Br. & Hatchuni & Asteraceae & Whole plant & Nasal problem \\
\hline 31. & $\begin{array}{l}\text { Clerodendrum indicum (L.) } \\
\text { Kuntze }\end{array}$ & Noli-gach & Verbenaceae & Roots & Stop bleeding \\
\hline 32. & Clerodendrum viscosum Vent. & Veck-gach & Verbenaceae & Leaves & Sores, diabetes \\
\hline 33. & Cnesmone javanica $\mathrm{Bl}$. & Chotta & Euphorbiaceae & Leaves & Snake bite, blood cancer \\
\hline 34. & Coccinia cordifolia Cogn. & Tela-kuchu & Cucurbitaceae & Whole plant & $\begin{array}{l}\text { Diabetes, burning } \\
\text { sensation }\end{array}$ \\
\hline 35. & Crotalaria pallida Ait. & $\begin{array}{l}\text { Kudugo- } \\
\text { jhunjhuni (1) }\end{array}$ & Fabaceae & Roots, leaves & $\begin{array}{l}\text { Stomach pain, urination } \\
\text { problem }\end{array}$ \\
\hline 36. & Curcuma caesia Roxb. & Kala-holod & Zingiberaceae & Rhizomes & Anti-poison, sore throat \\
\hline 37. & Curcuma longa L. & Holod & Zingiberaceae & $\begin{array}{l}\text { Flowers, } \\
\text { rhizomes }\end{array}$ & Blood purifier, tonic \\
\hline 38. & Cymbopogon citratus Stapf. & Dhan-sabarang & Poaceae & Roots, leaves & Cold, stomach ache \\
\hline 39. & Cyperus diffusus Vahl & Perazary & Cyperaceae & Leaves & Antiseptic \\
\hline 40. & Delima sarmentosa L. & Ulu-ludi & Dilleniaceae & Roots, leaves & Fever \\
\hline 41. & Desmodium triquitrum DC. & Komorsina & Fabaceae & Leaves & Paralysis \\
\hline 42. & $\begin{array}{l}\text { Diploclisia glaucescens (Bl.) } \\
\text { Diels }\end{array}$ & Sonattola & Menispermaceae & Leaves & Rheumatic pain \\
\hline 43. & $\begin{array}{l}\text { Dysophylla crassicaulis } \\
\text { Benth. }\end{array}$ & Shel-pata-richa & Lamiaceae & Leaves & $\begin{array}{l}\text { Menstrual problem (stop } \\
\text { bleeding) }\end{array}$ \\
\hline 44. & $\begin{array}{l}\text { Entada phaseoloides (L.) } \\
\text { Merr. }\end{array}$ & Gila & Fabaceae & Seeds & Poisoning, play game \\
\hline 45. & Eupatorium odoratum L. & Assam-pata & Asteraceae & Leaves & Wounds \\
\hline 46. & Ficus racemosa L. & Jagga-dumur & Moraceae & Fruits & Invigorative \\
\hline 47. & $\begin{array}{l}\text { Gomphostemma parviflorum } \\
\text { Wall. }\end{array}$ & $\begin{array}{l}\text { Kudugo- } \\
\text { jhunjhuni (2) }\end{array}$ & Lamiaceae & Roots & Irregular menstruation \\
\hline 48. & Hibiscus radiatus Cav. & Sorbo-amila & Malvaceae & Leaves & Jaundice \\
\hline 49. & Hibiscus sabdariffa L. & Amila & Malvaceae & Leaves & Catarrh \\
\hline 50. & $\begin{array}{l}\text { Hoya acuminata (Wight) } \\
\text { Benth. }\end{array}$ & Pasha-mash & Asclepiadaceae & Leaves & Ear diseases \\
\hline 51. & $\begin{array}{l}\text { Kalanchoe pinnata (Lamk.) } \\
\text { Pers. }\end{array}$ & Pathor -kuchi & Crassulaceae & Leaves & $\begin{array}{l}\text { Ear lesion, urination } \\
\text { problem }\end{array}$ \\
\hline 52. & $\begin{array}{l}\text { Lagenaria siceraria (Molina) } \\
\text { Standly }\end{array}$ & Kudugulo & Cucurbitaceae & Roots & Throat diseases \\
\hline 53. & Leea macrophylla Roxb. & Baggach & Leeaceae & Roots, leaves & Fracture, rheumatism \\
\hline 54. & Lygodium flexuosum Sw. & Kogti-jurgo & Lygodiaceae & Leaves & Sores \\
\hline 55. & Mangifera indica L. & Am & Anacardiaceae & Seeds & Diabetes, tonic \\
\hline
\end{tabular}


Table 1 contd.

\begin{tabular}{|c|c|c|c|c|c|}
\hline $\begin{array}{l}\text { Sl. } \\
\text { No. }\end{array}$ & Scientific name & Chakma name & Family & Part(s) used & $\begin{array}{l}\text { Disease(s) or symptom(s) } \\
\text { to be treated }\end{array}$ \\
\hline 56. & Melastoma malabathricum L. & $\begin{array}{l}\text { Moha-putting- } \\
\text { gulo }\end{array}$ & Melastomaceae & Roots & Stomach ache \\
\hline 57. & $\begin{array}{l}\text { Moghania macrophylla } \\
\text { Kuntze }\end{array}$ & $\begin{array}{l}\text { Kodorothang- } \\
\text { gach }\end{array}$ & Fabaceae & Leaves & Gastric \\
\hline 58. & Moringa oleifera Lamk. & Sajna & Moringaceae & Stems bark & Back ache, rheumatism \\
\hline 59. & Musa sapientum L. & Kola-gach & Musaceae & Leaves & Tumor \\
\hline 60. & Mussaenda glabra Vahl & $\begin{array}{l}\text { Bissollo- } \\
\text { karani/ Gach- } \\
\text { ranirtak }\end{array}$ & Rubiaceae & Whole plant & Menstrual problem \\
\hline 61. & Ocimum americanum L. & Sabarang & Lamiaceae & Whole plant & Stimulant \\
\hline 62. & Ocimum gratissimum L. & $\begin{array}{l}\text { Mitha- } \\
\text { phul/Ram-tulsi }\end{array}$ & Lamiaceae & Leaves & $\begin{array}{l}\text { Nasal diseases, skin } \\
\text { diseases }\end{array}$ \\
\hline 63. & Oroxylum indicum (L.) Vent. & Fona-gulogach & Bignoniaceae & Stem bark & Jaundice \\
\hline 64. & Oxalis corniculata $\mathrm{L}$. & Amrul & Oxalidaceae & Whole plant & Constipation \\
\hline 65. & Paederia foetida L. & Pada-bash-ludi & Rubiaceae & Leaves & Joint pain, rheumatism \\
\hline 66. & Peliosanthes teta Andrews & Dhub-melony & Liliaceae & Roots & Wounds \\
\hline 67. & Pentapetes phoenicea L. & $\begin{array}{l}\text { Dibuzza-phul- } \\
\text { gach }\end{array}$ & Sterculiaceae & Leaves & Boils \\
\hline 68. & $\begin{array}{l}\text { Phlogacanthus thyrsiflorus } \\
\text { N.E. }\end{array}$ & Vargi-nola & Acanthaceae & Leaves & Gout, rheumatism \\
\hline 69. & Phylanthus emblica L. & Amoloki & Euphorbiaceae & Fruits & Constipation \\
\hline 70. & Piper nigrum L. & Gul-morish & Piperaceae & Roots, leaves & $\begin{array}{l}\text { Fever, cough, catarrh, } \\
\text { rheumatism }\end{array}$ \\
\hline 71. & Plumbago indica L. & $\begin{array}{l}\text { Rangajat } \\
\text { aguna- teda }\end{array}$ & Plumbaginaceae & Roots & Stomach ache \\
\hline 72. & Plumbago zeylanica $\mathrm{L}$. & Chita-mul & Plumbaginaceae & Roots & Stomach ache \\
\hline 73. & Plumeria rubra L. & Bak-phul & Apocynaceae & Roots, stems & Blood cancer \\
\hline 74. & Polygonum flaccidum Meissn. & Biskatali & Polygonaceae & Leaves & $\begin{array}{l}\text { Sores and boils, } \\
\text { antiseptic }\end{array}$ \\
\hline 75. & Pothos scandens L. & Komorsina & Araceae & Stems, leaves & Fracture \\
\hline 76. & Premna esculenta Roxb. & Lalom-pata & Verbenaceae & Leaves & Appetizer \\
\hline 77. & Psidium guajava Batt & Goium & Myrtaceae & Leaves & Dysentery, toothache \\
\hline 78. & Pyrrosia piloselloides M.G. & Tenga-chara & Polypodiaceae & Leaves & Ear ache \\
\hline 79. & $\begin{array}{l}\text { Rauvolfia serpentina Benth. } \\
\text { ex Kurz }\end{array}$ & Surchan & Apocynaceae & Roots & $\begin{array}{l}\text { Blood pressure, stomach } \\
\text { ache }\end{array}$ \\
\hline 80. & Ricinus communis L & Varon pata & Euphorbiaceae & Leaves & $\begin{array}{l}\text { Boils, gynecological } \\
\text { problem }\end{array}$ \\
\hline 81. & Rubus hexagynus Roxb. & Kata-chola & Rosaceae & Roots & Fever \\
\hline 82. & Solanum myriacanthum Dun. & Karnafully & Solanaceae & Fruits & Aphrodisiac \\
\hline 83. & $\begin{array}{l}\text { Taebernaemontana divaricata } \\
\text { Bl. }\end{array}$ & Katto-dongor & Apocynaceae & Roots & Hiccup \\
\hline 84. & Terminalia bellerica Roxb. & Boragulo & Combretaceae & $\begin{array}{l}\text { Stems barks, } \\
\text { roots }\end{array}$ & $\begin{array}{l}\text { Blood dysentery, } \\
\text { urination problem }\end{array}$ \\
\hline 85. & Terminalia chebula Retz. & Hottail & Combretaceae & Fruits & $\begin{array}{l}\text { Blood dysentery, } \\
\text { stomach ache }\end{array}$ \\
\hline 86. & $\begin{array}{l}\text { Thevetia peruviana (Pers.) } \\
\text { Schum. }\end{array}$ & Goi-phul & Apocynaceae & Roots & Urination problem \\
\hline
\end{tabular}


Table 1 contd.

\begin{tabular}{|c|c|c|c|c|c|}
\hline $\begin{array}{l}\text { Sl. } \\
\text { No. }\end{array}$ & Scientific name & Chakma name & Family & Part(s) used & $\begin{array}{l}\text { Disease(s) or symptom(s) } \\
\text { to be treated }\end{array}$ \\
\hline 87. & $\begin{array}{l}\text { Typhonium trilobatum (L.) } \\
\text { Scott. }\end{array}$ & Harbaz & Araceae & Leaves & Rheumatism, body pain \\
\hline 88. & Uraria crinita (L.) Desv. & Bilai-langur & Fabaceae & Whole plant & Paralysis \\
\hline 89. & $\begin{array}{l}\text { Vernonia patula (Dryand.) } \\
\text { Merr. }\end{array}$ & Danta-utpal & Asteraceae & Roots & Stomach ache \\
\hline 90. & Zingiber zerumbet (L.) Sm. & Bhul-changa & Zingiberaceae & Rhizomes & Paralysis \\
\hline
\end{tabular}

\section{Acknowledgement}

The authors are grateful to the Ministry of Chittagong Hill Tracts Affairs, Government of Bangladesh for financial support to conduct the fieldwork.

\section{References}

Alam, M.K. 1992. Medical ethno-botany of the Marma tribe of Bangladesh. Economic Botany 46(3): 330330.

Alam, M.K., Choudhury, J. and Hassan, M.A. 1996. Some folk formularies from Bangladesh. Bangladesh J. Life Sci. 8(1): 49-63.

Chakma, S., Hossain, M.K., Khan, B.M. and Kabir, M.A. 2003. Ethno-botanical knowledge of Chakma community in the use of medicinal plants in Chittagong Hill Tracts, Bangladesh. MFP News XIII(3): 3-7.

Chowdhury, J., Alam, M.K. and Hassan, M.A. 1996. Some folk formularies against dysentery and diarrhea in Bangladesh. J. Econ. Taxon. Bot. Additional Series 12. Scientific Publishers, Jodhpur, pp. 20-23.

Ghani, A. 1998. Medicinal plants of Bangladesh: Chemical constituents and uses. Asiatic Society of Bangladesh, Dhaka, pp. 1-460.

Hassan, M.A. and Khan, M.S. 1986. Ethnobotanical record of Bangladesh-1: Plants used for healing fractured bones. J. Asiatic Soc. Bangladesh. (Sci.). 12(1\&2): 33-39.

Hassan, M.A. and Khan, M.A.1996. Ethnobotanical record of Bangladesh-2. Plants used for healing cuts and wounds. Bangladesh J. Plant Taxon. 3(2): 49-52.

Khaleque, K. 1995. Ethnic communities of Bangladesh. In: Gain, P. (ed.) Bangladesh, Land, Forest and Forest People, pp. 1-25. Society for Environment and Human Development (SEHD), Dhaka.

Khan, M.S., Hassan, M.A. and Uddin, M.Z. 2002. Ethnobotanical survey in Rema-Kalenga Wildlife Sanctuary (Habiganj) in Bangladesh. Bangladesh J. Plant Taxon. 9(1): 51-60.

Khisa, B. 1996. Chakma Talik Chikitsa. Herbal Medicine Centre Committee, Rajban Bihar, Rajbari, Rangamati, 1-136 pp.

Khisa, S.K. 1998. Ethnobotanical and cultural background of ethnic communities in forest resource management in Chittagong Hill Tracts. In: Banic, R.L., Alam, M.K., Pei, S.J. and Rastogi, A. (eds), Applied Ethno-botany, pp. 56-63. Bangladesh Forest Research Institute, Chittagong.

Rahman, M.A. 2003. Ethno-medico-botanical knowledge among tribals of Bangladesh. In: Ethnobotany and Medicinal Plants of Indian subcontinent, pp. 89-93. Scientific Publisher, Jodhpur. 
Rahman, M.A., Khisa, A., Uddin, S.B. and Wilcock, C.C. 2003. Indigenous knowledge of herbal medicine in Bangladesh - treatment of jaundice by the tribal communities of hill tracts districts. In: Sillitoe, P. (ed.), Indigenous Knowledge Development in Bangladesh - Present and Future, pp. 75-78.

Rahman, M.A. and Uddin, S.B. 1998. Some anti-rheumatic plants used by tribal people of Hill Tracts district. Biodiversity Newsletter, University of Chittagong 2(2): 4.

Rahman, M.A., Uddin, S.B. and Khisa, A. 1998. A report on some anti-jaundice plants from tribal community of Hill Tracts district. Biodiversity Newsletter, University of Chittagong 2(1): 4.

Mia, M.M.K. and Huq, A.M. 1988. A preliminary ethno-botanical survey in the Jointiapur, Tamabil and Jafflong area, Sylhet, Bangladesh National Herbarium Bull. 3: 1-10.

Tripura, S.L. 1994. Nature and Culture of the Chittagong Hill Tracts. Tribal Culture Institute, Rangamati Hill District, pp. 1-192.

Uddin, S.B. 2001. A comparative ethno botanical study among the tribal communities of Chittagong Hill Tracts, Bangladesh. PhD thesis, the University of Aberdeen, UK.

Uddin, M.Z., Hassan, M.A. and Sultana, M. 2006. Ethnobotanical survey of medicinal plants in Phulbari Upazila of Dinajpur District, Bangladesh. Bangladesh J. Plant Taxon. 12(1): 63-68.

Uddin, M.Z., Khan, M.S. and Hassan, M.A. 2001. Ethno medical plants records of Kalenga forest range (Habiganj), Bangladesh for malaria, jaundice, diarrhea and dysentery. Bangladesh J. Plant Taxon. 8(1): 101-104.

Uddin, S.N., Uddin, M.Z., Hassan, M.A. and Rahman, M.M. 2004. Preliminary ethno-medical plant survey in Khagrachari district, Bangladesh. Bangladesh J. Plant Taxon. 11(2): 39-48.

Yusuf, M., Choudhury, J.U., Wahab, M.A. and Begum, J. 1994. Medicinal plants of Bangladesh. Bangladesh Council of Scientific and Industrial Research, Dhaka, Bangladesh, pp. 1-340.

Yusuf, M., Rahman, M.A., Choudhury, J.U. and Begum, J. 2002. Indigenous knowledge about the use of Zingibers in Bangladesh. J. Econ. Taxon. Bot. 26(3): 566-570.

Yusuf, M., Wahab, M.A., Choudhury, J.U. and Begum, J. 2006. Ethno-medico-botanical knowledge from Kaulkhali proper and Betunia of Rangamati district. Bangladesh J. Plant Taxon. 13(1): 55-61.

(Manuscript received on 27 August 2007; revised on 18 November 2007) 